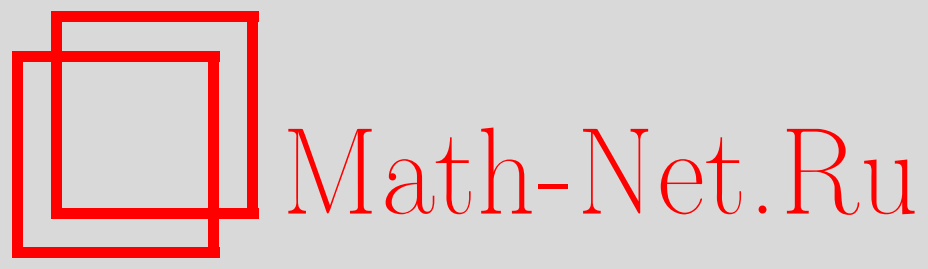

М. С. Агранович, А. М. Селицкий, Дробные степени операторов, отвечающих коэрцитивным задачам в липшицевых областях, Функи. анализ и его прил., 2013, том 47, выпуск 2, 2-17

DOI: https://doi.org/10.4213/faa3109

Использование Общероссийского математического портала Math$\mathrm{Net.Ru}$ подразумевает, что вы прочитали и согласны с пользовательским соглашением http://www. mathnet.ru/rus/agreement

Параметры загрузки:

IP : 3.82 .47 .9

26 апреля 2023 г., 18:01:53

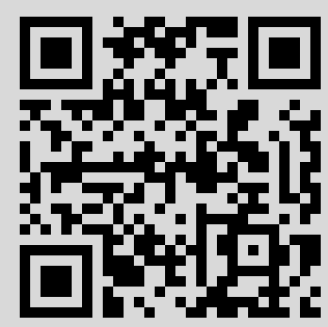


Функциональный анализ и его приложения

2013, т. 47, вып. 2, с. 2-17

УДК $517.98+517.95$

\title{
Дробные степени операторов, отвечающих коэрцитивным задачам в липшицевых областях ${ }^{*}$
}

\author{
(c) 2013. М. С. АГРАнович, А. М. СЕЛицКий
}

\begin{abstract}
Пусть $\Omega$ - ограниченная липшицева область в $\mathbb{R}^{n}, n \geqslant 2$, и пусть в ней задан матричный сильно эллиптический оператор $L$ 2-го порядка, записанный в дивергентной форме. Обширная литература посвящена изучению областей определения дробных степеней операторов, отвечающих задачам для уравнения $L u=f$, прежде всего Дирихле и Неймана, с однородными граничными условиями, включая решение проблемы Като, возникшей в 1961 г. Охвачены также смешанные задачи и некоторый класс задач для систем высших порядков.

Мы предлагаем новый абстрактный подход к этой проблематике, позволяющий существенно проще и единым образом получить наиболее важные, с нашей точки зрения, результаты и охватить новые операторы - классические граничные операторы на липшицевой границе Г области $\Omega$ или ее части. Для этого мы одновременно рассматриваем два хорошо известных оператора, сопоставляемых граничной задаче.
\end{abstract}

Оператор $L$ имеет вид

$$
L=-\sum_{j, k=1}^{n} \partial_{j} a_{j, k}(x) \partial_{k}+\sum_{j=1}^{n} b_{j}(x) \partial_{j}+c(x)
$$

где $\partial_{j}=\partial / \partial x_{j}$ и коэффициенты $-d \times d$-матрицы из комплекснозначных функций. Эти коэффициенты подчиняются некоторым слабым предположениям о гладкости (см. п. 5). Условие сильной эллиптичности [10] состоит в том, что

$$
\operatorname{Re} \sum a_{j, k}(x) \xi_{j} \xi_{k} \geqslant C_{0}|\xi|^{2} I
$$

при $\xi \in \mathbb{R}^{n}$ и $x \in \bar{\Omega}$ в смысле неравенства для квадратичных форм, где $C_{0}$ - положительная постоянная. С этим оператором связана «энергетическая» форма

$\Phi_{\Omega}(u, v)=\int_{\Omega}\left[\sum a_{j, k}(x) \partial_{k} u(x) \cdot \partial_{j} \overline{v(x)}+\sum b_{j}(x) \partial_{j} u(x) \cdot \overline{v(x)}+c(x) u(x) \cdot \overline{v(x)}\right] d x$.

Наша основная цель в этой статье - объяснение простого и единого подхода к положительному решению проблемы Като в наиболее интересных для нас вариантах; мы напомним ее в п. 3. Этот подход имеет и новые приложения.

Наши основные результаты - это теоремы в п. 3. Сначала мы будем рассматривать как модельные только задачи Дирихле и Неймана для системы $L u=f$ в ограниченных липшицевых областях. Но изложение будет сразу абстрактным,

*Работа поддержана грантами РФФИ 11-01-00277, 12-01-00523а и 13-01-00923. 
а в п. 4 мы объясним применимость нашего подхода к смешанным задачам, задачам для общих сильно эллиптических систем высших порядков и к новому в этой тематике (насколько мы знаем) классу операторов - классическим граничным операторам на липшицевой поверхности. При этом не нужна перестройка нашей схемы рассуждений. Некоторые известные несложные импликации будут заимствованы из работ по рассматриваемой тематике. Краткое сравнение нашей схемы с методикой в других работах будет приведено в п. 5.

\section{1. Два классических операторных подхода к коэрцитивным задачам} для сильно эллиптических систем. Рассмотрим следующую абстрактную ситуацию. Пусть заданы три (сепарабельных) гильбертовых пространства $H_{-1}$, $H_{0}, H_{1}$ с компактными (для простоты) и плотными вложениями

$$
H_{-1} \supset H_{0} \supset H_{1} .
$$

Скалярные произведения и нормы в этих пространствах обозначим через $(\cdot, \cdot)_{k}$ и $\|\cdot\|_{k}, k=-1,0,1$. Предположим, что пространства $H_{-1}$ и $H_{1}$ дуальны относительно формы $(u, v)_{0}$ и что $H_{0}$ получается из $H_{-1}$ и $H_{1}$ комплексной интерполяцией (см. определения в [9] и [17]) с индексом $1 / 2$ :

$$
H_{0}=\left[H_{-1}, H_{1}\right]_{1 / 2} \text {. }
$$

Далее, предположим, что на $H_{1} \times H_{1}$ задана ограниченная полуторалинейная форма $\Phi(u, v)$, удовлетворяющая неравенству сильной коэриитивности

$$
\|u\|_{1}^{2} \leqslant C \operatorname{Re} \Phi(u, u)
$$

с некоторой постоянной $C>0$. Тогда соотношение

$$
\Phi(u, v)=\left(A_{1} u, v\right)_{0} \quad\left(u, v \in H_{1}\right)
$$

определяет ограниченный линейный оператор $A_{1}: H_{1} \rightarrow H_{-1}$, и этот оператор обратим по теореме Лакса-Мильграма (см., например, [13, гл. 2, теорема 9.1], [48], [49] или [7]). Это модель первого подхода к интересующим нас задачам с однородными граничными условиями.

В случае сильно эллиптической системы 2-го порядка пространство $H_{0}-$ это $L_{2}(\Omega)$. В задаче Дирихле $H_{1}=\widetilde{H}^{1}(\Omega), H_{-1}=H^{-1}(\Omega)$, в задаче Неймана $H_{1}=H^{1}(\Omega), H_{-1}=\widetilde{H}^{-1}(\Omega)$. Коэрцитивность (неравенство вида (1.3) с добавлением $\|u\|_{0}^{2}$ справа) следует из сильной эллиптичности в случае матричной задачи Дирихле и скалярной $(d=1)$ задачи Неймана при $a_{j, k}=a_{k, j}$, но это дополнительное предположение в случае матричной задачи Неймана (хорошо известны достаточные условия, см. [49], [48] или [7]).

Здесь $H^{s}(\Omega)(s \in \mathbb{R})$ - это $L_{2}$-пространство бесселевых потенциалов в $\Omega$ - сужений элементов из $H^{s}\left(\mathbb{R}^{n}\right)$ на $\Omega$ с обычной inf-нормой. При $s \geqslant 0$ это пространства $W_{2}^{s}$ Соболева-Слободецкого. Через $\widetilde{H}^{s}(\Omega)$ обозначается подпространство в $H^{s}\left(\mathbb{R}^{n}\right)$, состоящее из элементов с носителями в $\bar{\Omega}$. При $|s|<1 / 2$ пространства $H^{s}(\Omega)$ и $\widetilde{H}^{s}(\Omega)$ можно отождествить. При не полуцелом $s \geqslant 0$ пространство $\widetilde{H}^{s}(\Omega)$ можно отождествить с $\stackrel{\circ}{H}^{s}(\Omega)$ - замыканием в $H^{s}(\Omega)$ линеала $C_{0}^{\infty}(\Omega)$. При любом $s$ пространства $\widetilde{H}^{s}(\Omega)$ и $H^{-s}(\Omega)$ дуальны относительно продолжения скалярного произведения в $L_{2}(\Omega)$ на их прямое произведение. Добавим, что пространства $H^{s}(\Gamma)$ на липшицевой границе Г области $\Omega$ определяются при помощи локальных карт при $|s| \leqslant 1$. См. [48] или [7]. 
Та же форма $\Phi$ определяет сопряженный к $A_{1}$ оператор $A_{1}^{*}$ равенством

$$
\Phi(u, v)=\left(u, A_{1}^{*} v\right)_{0} \quad\left(u, v \in H_{1}\right),
$$

или, что то же,

$$
\overline{\Phi(v, u)}=\left(A_{1}^{*} u, v\right)_{0} \quad\left(u, v \in H_{1}\right) .
$$

Операторы $A_{1}$ и $A_{1}^{*}$ формально сопряжены относительно формы $(u, v)_{0}$ : на элементах из $H_{1}$. В наших модельных задачах $A_{1}^{*}$ отвечает формально сопряженному к $L$ оператору $L^{*}$.

Пусть $\Lambda=\Lambda_{\theta}$ - наименьший замкнутый угол (сектор) раствора $2 \theta$ с биссектрисой $\mathbb{R}_{+}$, содержащий все значения формы $\Phi(u, u)$. Из (1.3) следует, что мнимая часть формы оценивается через вещественную, поэтому $2 \theta<\pi$. Оператор $A_{1}$ в $H_{-1}$ имеет область определения $H_{1}$ и дискретный спектр, содержащийся в этом угле; при этом собственных значений нет в некоторой окрестности начала. То же верно для оператора $A_{1}^{*}$. Корневые векторы лежат в $H_{1}$.

Пусть теперь форма $\Phi(u, v)$ эрмитова:

$$
\Phi(u, v)=\overline{\Phi(v, u)} \quad\left(u, v \in H_{1}\right) .
$$

Тогда сектор $\Lambda$ - это просто луч $\overline{\mathbb{R}}_{+}$. Переобозначим форму в этом случае через $\Psi(u, v)$ и соответствующий оператор через $S_{1}$. Он обратим и является формально самосопряженным относительно формы $(u, v)_{0}:\left(S_{1} u, v\right)_{0}=\left(u, S_{1} v\right)_{0}$ при $u, v \in H_{1}$. Эрмитова форма

$$
\langle u, v\rangle_{1}=\Psi(u, v)=\left(S_{1} u, v\right)_{0}=\left(u, S_{1} v\right)_{0}
$$

на $H_{1}$ удовлетворяет условию (сильной) коэрцитивности

$$
\|u\|_{1}^{2} \leqslant C\langle u, u\rangle_{1} \quad\left(u \in H_{1}\right),
$$

и мы можем принять ее за скалярное произведение в $H_{1}$. Соответствующая норма эквивалентна исходной норме в $H_{1}$. Заменяя $u$ на $S_{1}^{-1} u$ и $v$ на $S_{1}^{-1} v$, получаем новое скалярное произведение в $H_{-1}$

$$
\langle u, v\rangle_{-1}=\left(S_{1}^{-1} u, v\right)_{0}=\left(u, S_{1}^{-1} v\right)_{0} .
$$

Соответствующая норма эквивалентна исходной норме в $H_{-1}$, а оператор $S_{1}$ (с областью определения $H_{1}$ ) является самосопряженным в $H_{-1}$ относительно этого скалярного произведения. Компактный оператор $S_{1}^{-1}$ в $H_{-1}$ тоже является самосопряженным относительно этого скалярного произведения.

В общем случае, поскольку операторы $A_{1}$ и $A_{1}^{*}$ в $H_{-1}$ имеют общую область определения $H_{1}$, вещественная часть $S_{1}$ этих операторов легко вводится как их полусумма. Соответствующая форма $\Psi(u, v)$ есть полусумма форм $\Phi(u, v)$ и $\overline{\Phi(v, u)}$, она при $u=v$ положительна и удовлетворяет неравенству (1.9):

$$
\|u\|_{1}^{2} \leqslant C \Psi(u, u) \quad\left(u \in H_{1}\right) .
$$

По оператору $S_{1}$ определяются скалярные произведения (1.8) и (1.10). Фактически он строится по форме $\Phi$.

Следующее предложение известно и легко проверяется.

Предложение 1.1. Пусть $\Lambda^{\prime}-$ открытый угол с вершиной в начале, содержащий $\Lambda \backslash\{0\}$. Тогда для решения $v(\lambda)$ уравнения $\left(A_{1}-\lambda I\right) v(\lambda)=f$ при $f \in H_{-1}, \lambda \in \mathbb{C} \backslash \Lambda^{\prime}$ справедлива априорная оченка

$$
\|v(\lambda)\|_{1}+|\lambda|\|v(\lambda)\|_{-1} \leqslant C_{1}\|f\|_{-1}
$$


с постоянной $C_{1}$, не зависящей от $f u \lambda$.

Поясним, что при $|\arg \lambda| \geqslant \pi / 2$ сначала получается равномерная по $\lambda$ оценка для $\|v(\lambda)\|_{1}$ из (1.3)-(1.4), так как справа в (1.3) можно прибавить $-\operatorname{Re} \lambda(u, u)_{0}$. Затем используем уравнение для $v(\lambda)$. На все нужные $\lambda$ оценка распространяется переходом от формы $\Phi$ к формам $e^{i \theta} \Phi$ с малыми $|\theta|$.

Bторой подход к нашим задачам состоит в том, что по существу то же, что и (1.4), соотношение

$$
\Phi(u, v)=\left(A_{2} u, v\right)_{0} \quad\left(v \in H_{1}\right)
$$

рассматривается как определяющее неограниченный линейный оператор $A_{2}$ в $H_{0}$ с областью определения $H_{2}=D\left(A_{2}\right)$, содержащейся в $H_{1}$ :

$$
H_{1} \supset H_{2} \text {. }
$$

В абстрактных рамках область определения $D\left(A_{2}\right)$ оператора $A_{2}$ определяется как состоящая из таких $u \in H_{1}$, что антилинейный функционал $\Phi(u, v)$ на $H_{1}$ непрерывен по норме $\|v\|_{0}$ :

$$
|\Phi(u, v)| \leqslant C_{u}\|v\|_{0}
$$

Он поэтому продолжается до непрерывного антилинейного функционала на $H_{0}$ и записывается в виде (1.13). В $\mathrm{H}_{2}$ вводится норма графика

$$
\|u\|_{2}=\left(\|u\|_{0}^{2}+\left\|A_{2} u\right\|_{0}^{2}\right)^{1 / 2},
$$

после чего $H_{2}$ становится гильбертовым пространством, а $A_{2}$ - ограниченным оператором из $\mathrm{H}_{2}$ в $H_{0}$.

Если $A_{1} u=f \in H_{0}$, то форма $\Phi(u, v)=(f, v)_{0}$, конечно, непрерывна по $v$ в $H_{0}$, так что $u \in H_{2}, A_{2} u=A_{1} u, A_{2}^{-1} f=A_{1}^{-1} f$. Поэтому $A_{2}$ есть сужение onepamopa $A_{1}$ на $H_{2}$. В задачах Дирихле и Неймана это реализации соответствующего оператора $L$ в $L_{2}(\Omega)$.

Оператор $A_{2}^{*}$, сопряженный к $A_{2}$ в $H_{0}$, и самосопряженный оператор $S_{2}$ определяются соответственно формой $\overline{\Phi(v, u)}$ и полусуммой $\Psi(u, v)$ форм $\Phi(u, v)$ и $\overline{\Phi(v, u)}$. Эти операторы соответственно совпадают с $A_{1}^{*}$ на $D\left(A_{2}^{*}\right)$ и с $S_{1}$ на $D\left(S_{2}\right)$.

Предложение 1.2. Oператор $A_{2}$ обратим. То же верно для операторов $A_{2}^{*}$ $u S_{2}$.

Доказательство см., например, в [42, с. 11].

Следствие 1.3. Пространство $\mathrm{H}_{2}=D\left(A_{2}\right)$ плотно и компактно вложено в $H_{1}$. То же верно для $D\left(A_{2}^{*}\right)$ и $D\left(S_{2}\right)$.

Действительно, соответствующий оператор вложения $\mathscr{E} 2,1$ можно представить в виде $A_{1}^{-1} \mathscr{E}_{0,-1} A_{2}$, где $\mathscr{E}_{0,-1}$ - оператор вложения $H_{0}$ в $H_{-1}$. Последний компактен, поэтому $\mathscr{E}_{2,1}$ компактен. Далее, если $u_{0} \in H_{1}$, то $v_{0}=A_{1} u_{0}$ можно аппроксимировать в $H_{-1}$ элементами $v_{k} \in H_{0}$. Тогда $w_{k}=A_{2}^{-1} v_{k}=A_{1}^{-1} v_{k}$ принадлежат $H_{2}$ и сходятся к $u_{0}$ в $H_{1}$.

См. также [11, гл. VI, §2].

Для оператора $A_{2}$ справедлив также аналог предложения 1.1: 
Предложение 1.4. Для решений $v(\lambda)$ в $H_{2}$ уравнения $\left(A_{2}-\lambda I\right) v(\lambda)=f$, $f \in H_{0}$, при $\lambda \in \mathbb{C} \backslash \Lambda^{\prime}$ справедлива оченка

$$
\|v(\lambda)\|_{2}+|\lambda|\|v(\lambda)\|_{0} \leqslant C_{2}\|f\|_{0}
$$

c постоянной $C_{2}$, не зависящей от $f$ u $\lambda$. То же верно для операторов $A_{2}^{*} u$ $\mathrm{S}_{2}$ вместо $\mathrm{A}_{2}$.

Здесь сначала оцениваем $|\operatorname{Re} \lambda|\|v(\lambda)\|_{0}$ через $\|f\|_{0}$ при $\operatorname{Re} \lambda \leqslant 0$. В немного более узком угле с биссектрисой $\mathbb{R}_{-}$получаем отсюда оценку для $|\lambda|\|v(\lambda)\|_{0}$. На угол раствора больше $\pi / 2$ она распространяется умножением оператора и формы на $e^{i \theta}$. Теперь из уравнения получается оценка для $\left\|A_{2} v(\lambda)\right\|_{0}$. Это приводит к оценке первого слагаемого в (1.17).

Спектры и корневые векторы у операторов $A_{1}$ и $A_{2}$ одинаковы. Это следует из того, что собственные векторы оператора $A_{1}$ принадлежат $H_{0}$, а значит, $H_{2}$, поскольку собственные значения ненулевые. Аналогично обстоит дело с операторами $A_{2}^{*}$ и $A_{1}^{*}$, а также с операторами $S_{2}$ и $S_{1}$.

Второй подход к задачам для сильно эллиптических систем тоже общеизвестен, отражен, например, в монографиях [42] и [11] (гл. VI, §2) и рассматривался в очень многих работах. Важнейший вопрос - возможно более полное описание пространства $D\left(A_{2}\right)=H_{2}$. Для систем 2-го порядка с гладкими коэффициентами в области с гладкой границей оно получается, если выполнены условия эллиптичности задачи, из результатов общей теории эллиптических задач (см., например, [18] или [7]). Это подпространство в $H^{2}(\Omega)$, выделяемое однородными граничными условиями. При этом ясно, что, например, в случае задачи Неймана области определения операторов $A_{2}$ и $A_{2}^{*}$ могут быть разными. В области с липшицевой границей в общем случае $D\left(A_{2}\right)$ неизвестна.

Первый подход очень удобен «адекватностью» используемых и заранее заданных пространств рассматриваемым задачам. В наших конкретных задачах это пространства типа Соболева-Слободецкого. Операторы $A_{1}$ и $A_{1}^{*}$ имеют общую и известную область определения $H_{1}$. Второй подход удобен тем, что правые части системы принадлежат $L_{2}(\Omega)$.

Оба подхода обобщаются на ситуацию, в которой оператор фредгольмов и приводится к обратимому включением в $L$ слагаемого $\mu u(x)$ с достаточно большим положительным $\mu$.

2. Позитивные операторы и их дробные степени. Напомним некоторые общеизвестные определения и факты. Пусть $X$ - банахово пространство с нормой $\|\cdot\|$ и $A-$ замкнутый линейный оператор в $X$ с плотной областью определения $D(A) \subset X$. Он называется позитивным, если замкнутая отрицательная полуось $\overline{\mathbb{R}}_{-}$принадлежит резольвентному множеству этого оператора и на ней для резольвенты $R(\lambda)=(A-\lambda I)^{-1}$ выполняется неравенство

$$
\|R(\lambda)\| \leqslant C(1+|\lambda|)^{-1} .
$$

Отсюда следует, что резольвентное множество содержит некоторый сектор с биссектрисой $\mathbb{R}_{-}$и некоторую окрестность начала координат (см., например, [12]); в угле чуть меньшего раствора с той же биссектрисой и чуть меньшей окрестности сохраняется неравенство вида (2.1). Для простоты будем считать, что $A$ имеет дискретный спектр.

Через $\Lambda$ сейчас обозначим фиксированный замкнутый сектор с биссектрисой $\mathbb{R}_{+}$, вне которого верна оценка (2.1). Он содержит спектр оператора. 
Примерами позитивных операторов являются оператор $A_{1}$ в $H_{-1}$ и оператор $A_{2}$ в $H_{0}$ из п. 1. В этих случаях есть общий для них сектор $\Lambda$ раствора меньше $\pi$. Примеры позитивных операторов в общей теории «гладких» эллиптических задач - это эллиптические с параметром вдоль $\mathbb{R}$ - системы и задачи с однородными граничными условиями, однозначно разрешимые во всех точках замыкания этого луча (см. [21] или [8]).

Для позитивного оператора $A$ можно определить его степени $A^{z}$ с произвольным комплексным $z$. Степени $A^{-\alpha}$ с $\operatorname{Re} \alpha>0$ определяются формулой

$$
A^{-\alpha}=-\frac{1}{2 \pi i} \int_{l} \lambda^{-\alpha} R(\lambda) d \lambda .
$$

Здесь бесконечный контур $l$ охватывает спектр оператора $A$. В основном этот контур идет по лучам, охватывающим сектор $\Lambda$, но вблизи начала координат обходит его справа и не имеет общих точек с $\overline{\mathbb{R}}$. . Направление обхода - сверху из бесконечности, т. е. положительное. Это ограниченный оператор. Неограниченный оператор $A^{\alpha}$ определяется как обратный к $A^{-\alpha}$. Определения степеней $A^{\alpha}$ при общих комплексных $\alpha$ можно смотреть, например, в книгах [17] и [12], а также в [22] и [35].

При перемножении двух степеней действует обычное правило сложения показателей. Если $0 \leqslant \alpha<\beta$, то область определения $D\left(A^{\beta}\right)$ плотно вложена в $D\left(A^{\alpha}\right)$. Кроме того, в гильбертовом пространстве $\left(A^{\alpha}\right)^{*}=\left(A^{*}\right)^{\alpha}$ (см., например, [35]).

В литературе большое внимание было уделено нахождению областей определения положительных дробных степеней, в частности, из-за их роли в нестационарных задачах. Следующая теорема доказана в книге Трибеля [17, п. 1.15].

Теорема 2.1. Пусть $A$ - позитивный оператор в гилъбертовом пространстве $X=H$ и его чисто мнимые степени $A^{\text {it }}$ - ограниченные операторь при достаточно малом $t$, имеющие равномерно по $t$ ограниченные нормы. Тогда если $0 \leqslant \operatorname{Re} \alpha<\operatorname{Re} \beta<\infty u 0<\theta<1$, mo

$$
\left[D\left(A^{\alpha}\right), D\left(A^{\beta}\right)\right]_{\theta}=D\left(A^{\alpha(1-\theta)+\beta \theta}\right) .
$$

Арендт в [22, п. 4.4.10] указывает, что верно и обратное утверждение: из (2.3) следует локальная по $t$ равномерная ограниченность операторов $A^{i t}$ с вещественным $t$.

Условие локальной ограниченности чисто мнимых степеней выполнено не всегда, см., например, [41, с. 342].

Простейший случай, когда чисто мнимые степени ограничены, - это случай самосопряженного позитивного оператора $A$ в сепарабельном гильбертовом пространстве $H$. Если спектр этого оператора дискретен, то в $H$ имеется ортонормированный базис из собственных векторов $e_{j}$ нашего оператора $(j=1,2, \ldots)$, и если $\lambda_{j}-$ соответствующие собственные значения, то любой вектор в $H$ разлагается в ряд

$$
u=\sum_{1}^{\infty}\left(u, e_{j}\right) e_{j}, \quad\|u\|^{2}=\sum_{1}^{\infty}\left|\left(u, e_{j}\right)\right|^{2},
$$


а оператор $A^{\alpha}$ действует по формуле

$$
A^{\alpha} u=\sum_{1}^{\infty} \lambda_{j}^{\alpha}\left(u, e_{j}\right) e_{j}
$$

Этот оператор при $\operatorname{Re} \alpha>0$ является неограниченным: ряд

$$
\left\|A^{\alpha} u\right\|^{2}=\sum\left|\lambda_{j}^{\alpha}\right|^{2}\left|\left(u, e_{j}\right)\right|^{2}
$$

сходится не при всех $u$. Но очевидно, что при чисто мнимом $\alpha=i t$ это равномерно по $t$ ограниченный оператор.

Трибель пишет, что об ограниченности чисто мнимых степеней позитивных операторов мало что известно. Однако для операторов, отвечающих «гладким» задачам, эллиптическим с параметром вне угла раствора меньше $\pi$ (а также «бисекториальным» задачам, эллиптическим с параметром вне вертикальных углов, содержащих мнимую ось), положительный результат содержится в работе Сили [51].

Глубокие исследования проблемы Като принесли положительные результаты для операторов $A_{1}$, отвечающих, в частности, коэрцитивным задачам Дирихле и Неймана в липшицевых областях. Мы напомним эту проблему и объясним наш подход к ней в следующем пункте.

Для подготовки к этому приведем результат Лионса [43]-Яги [55].

Теорема 2.2. Пусть для позитивного оператора $A$ в гильбертовом пространстве $Н$ выполнено соотношение

$$
D\left(A^{\alpha}\right)=D\left(A^{* \alpha}\right)
$$

при $0<\alpha<\varepsilon$ с достаточно малым $\varepsilon$. Тогда

$$
D\left(A^{\alpha}\right)=[H, D(A)]_{\alpha}, \quad D\left(A^{* \alpha}\right)=\left[H, D\left(A^{*}\right)\right]_{\alpha} \quad(0 \leqslant \alpha \leqslant 1) .
$$

При этом чисто мнимые степени $A^{\text {it }}$ оператора $A$ локально ограничень.

Упомянем тесную связь позитивных операторов с полугруппами, важную при рассмотрении нестационарных задач. См., например, [12], [17], [22], [35].

3. Дробные степени операторов, отвечающих коэрцитивным задачам в липшицевых областях, и проблема Като. Вернемся к операторам из п. 1. Будем считать, что выполнены указанные там предположения о пространствах и операторах. Но при обсуждении проблемы Като мы дополним их предположением, сформулированным немного ниже.

Области определения дробных степеней $S_{1}^{\alpha}$ самосопряженного оператора $S_{1}$, как видно из сказанного в предыдущем пункте, при $0 \leqslant \alpha \leqslant 1$ образуют шкалу для комплексного метода интерполяции между пространствами $H_{-1}$ и $H_{1}$. Обозначим эти пространства через $H_{\tau}, \tau=-1+2 \alpha, \alpha \in[0,1]$. Поясним, что если $\left\{e_{j}\right\}_{1}^{\infty}$ - ортонормированный базис из собственных векторов оператора $S_{1}$ в $H_{-1}$ и $\lambda_{j}$ - соответствующие собственные значения, то $H_{\tau}$ состоит из векторов

$$
u=\sum_{1}^{\infty} \lambda_{j}^{\alpha}\left\langle u, e_{j}\right\rangle_{-1} e_{j} \quad \text { c }\|u\|_{\tau}^{2}=\sum_{1}^{\infty} \lambda_{j}^{2 \alpha}\left|\left\langle u, e_{j}\right\rangle_{-1}\right|^{2}<\infty .
$$

При $\tau=0$ оно совпадает с $H_{0}$. Оператор $S_{1}^{\alpha}$ действует ограниченным и обратимым образом из $H_{\tau_{1}}$ в $H_{\tau_{2}}$ при $\tau_{2}=\tau_{1}-2 \alpha$. 
Эту шкалу можно, используя степени оператора $S_{1}$, продолжить за точки \pm 1 . В задачах Дирихле и Неймана в липшицевых областях, как мы отметили в п. 1, это пространства $H^{\tau}(\Omega)$ и $\widetilde{H}^{\tau}(\Omega)$, заданные априорно. В частности, $S_{1}$ остается ограниченным и обратимым оператором из $H_{1+\gamma}$ в $H_{-1+\gamma}$ при любом $\gamma \in \mathbb{R}$. Но мы договоримся пользоваться обозначением $H_{\tau}$ только при $\tau \in$ $(-3 / 2,3 / 2)$, чтобы сохранить обозначение $H_{2}$ для $D\left(A_{2}\right)$. В гладком случае в этих обозначениях нет противоречий и можно рассматривать $H_{\tau}$ при любых $\tau$.

Мы предположим выполненным следующее

Условие 3.1. Операторь $A_{1}$ u $A_{1}^{*}$ остаются ограниченными из $H_{1+\gamma}$ в $H_{-1+\gamma}$ при $|\gamma|<\varepsilon$ с некоторым малым $\varepsilon>0$.

В задачах Дирихле и Неймана с этим нет проблем (см. [1] или [7]). Такая ограниченность обеспечивается предположением о небольшой гладкости коэффициентов.

Более того, по теореме Шнейберга об экстраполяции обратимости оператоpa, действующего ограниченным образом из одной интерполяционной шкалы в другую (см. [20] или [7]), эти операторы обратимы при $|\gamma|<\varepsilon_{1}$, где $\varepsilon_{1}-$ некоторое достаточно малое положительное число, $\varepsilon_{1} \leqslant \varepsilon$. Для коэрцитивных задач Дирихле и Неймана это теоремы о гладкости решений (см. [1], [2], [7]).

В этом пункте мы объясним предлагаемый нами простой путь к важным результатам для дробных степеней операторов $A_{1}$ и $A_{2}$. Начнем с оператора $A_{2}$.

Как мы уже отмечали, в общем случае нет информации о $H_{2}=D\left(A_{2}\right)$. Однако Като из элементарных соображений получил, даже для позитивных операторов с углом $\Lambda_{\theta}$ раствора $2 \theta<\pi([39$, теорема 1.1$])$, что

$$
D\left(A_{2}^{\alpha}\right)=D\left(A_{2}^{* \alpha}\right)=D\left(S_{2}^{\alpha}\right) \quad \text { при } 0 \leqslant \alpha<1 / 2 .
$$

Таких равенств, как отметил Като, в общем случае нет при $1 / 2<\alpha<1$, а Лионс показал (на том же примере) [43], что их может не быть и при $\alpha=1 / 2$. (См. также контрпример Макинтоша в [44] с не дифференциальным оператором, отвечающим коэрцитивной форме.) Возникла содержательная проблема: когда справедливы равенства

$$
D\left(A_{2}^{1 / 2}\right)=D\left(A_{2}^{* 1 / 2}\right)=H_{1} .
$$

Она получила известность как проблема Kamo (the Kato square root problem) и вызвала много содержательных исследований. В частности, для задач Дирихле и Неймана и других упомянутых в аннотации задач в липшицевых областях разными и непростыми способами было показано, что проблема решается положительно. См. ниже ссылки в п. 4 и п. 5.

Заметим, что в силу теоремы 2.2

$$
D\left(A_{2}^{\alpha}\right)=\left[H_{0}, D\left(A_{2}\right)\right]_{\alpha}, \quad D\left(A_{2}^{* \alpha}\right)=\left[H_{0}, D\left(A_{2}^{*}\right)\right]_{\alpha} \quad(0 \leqslant \alpha \leqslant 1) .
$$

Но $D\left(A_{2}\right)$ и $D\left(A_{2}^{*}\right)$ в общем случае остаются не известными и могут не совпадать, так что решение проблемы Като непосредственно отсюда не следует.

Для гладких эллиптических задач пространства справа в (3.4) описаны в работе Гривара [34], см. также Сили [52], и из этого описания видно, что проблема в этом случае решается положительно.

Совпадение двух из пространств (3.3) влечет совпадение всех трех ([40], [43]). Поэтому, в частности, проблема решается положительно в случае самосопряженного оператора $S_{2}$. 
Мы хотим теперь показать, что проблема Като решается положительно в принятой нами общности.

Теорема 3.2. При наших предположениях для операторов $A_{2}$ u $A_{2}^{*}$ справедливы равенства (3.3).

Доказательство. Положим

$$
B=S_{1}^{-1 / 2} A_{1} S_{1}^{-1 / 2} .
$$

Это при наших предположениях ограниченный обратимый оператор в $H_{\gamma}$ при малых $|\gamma|$, и мы имеем существенную для дальнейшего факторизацию операmopa $A_{1}$ :

$$
A_{1}=S_{1}^{1 / 2} B S_{1}^{1 / 2}
$$

Оператор $B$ не произволен: так как $S_{1}=\frac{1}{2}\left[A_{1}+A_{1}^{*}\right]$, то

$$
A_{1}^{*}=2 S_{1}-A_{1}=2 S_{1}-S_{1}^{1 / 2} B S_{1}^{1 / 2}=S_{1}^{1 / 2}(2 I-B) S_{1}^{1 / 2}=S_{1}^{1 / 2} B^{*} S_{1}^{1 / 2}
$$

и

$$
\frac{1}{2}\left(B+B^{*}\right)=I
$$

Лемма 3.3. Имеют место непрерывные вложения

$$
H_{1} \subset D\left(A_{2}^{1 / 2}\right), \quad H_{1} \subset D\left(A_{2}^{* 1 / 2}\right) .
$$

Отсюда теорема 3.2 следует в силу результата из [40, с. 293]; см. также [43]. Этот путь подсказан нам работой [32], в которой рассмотрены более специальные задачи. В отличие от нее мы будем пользоваться оператором $A_{1}$, совпадающим с $A_{2}$ на $D\left(A_{2}\right)$, и его факторизацией (3.6).

Пусть $\gamma$ - малое положительное число. Тогда $D\left(A_{2}\right) \subset H_{1+\gamma}$, так как оператор $A_{1}$ взаимно однозначно и непрерывно отображает эти пространства на $H_{0} \subset H_{-1+\gamma}$. Последнее включение является плотным, поэтому $D\left(A_{2}\right)$ плотно вложено в $H_{1+\gamma}$.

Для доказательства леммы 3.3, как в [32], докажем следующую лемму.

Лемма 3.4. Пространство $H_{1+\gamma}$ непрерывно вложено в пространства $D\left(A_{2}^{(1+\gamma) / 2}\right)$ и $D\left(A_{2}^{*(1+\gamma) / 2}\right)$.

Доказательство леммы 3.4. Зафиксируем любые $u$ и $v$ сначала в $D\left(A_{2}\right)$ и $D\left(A_{2}^{*}\right)$ соответственно. Положим $\delta=(1-\gamma) / 2$. Тогда $\delta \in(0,1 / 2)$.

Оператор $A_{2}^{*-\delta}$ непрерывен из $H_{0}$ в $D\left(A_{2}^{* \delta}\right)$. Но в силу $(3.2)$ и самосопряженности оператора $S_{2}$

$$
D\left(A_{2}^{* \delta}\right)=D\left(S_{2}^{\delta}\right)=\left[H_{0}, D\left(S_{2}^{1 / 2}\right)\right]_{2 \delta}=\left[H_{0}, H_{1}\right]_{2 \delta}=H_{2 \delta} .
$$

Поэтому $A_{2}^{*-\delta}-$ непрерывный оператор из $H_{0}$ в $H_{2 \delta}$. Так как $2 \delta-1=-\gamma$, то $S_{1}^{1 / 2} A_{2}^{*-\delta}-$ непрерывный оператор из $H_{0}$ в $H_{-\gamma}$ :

$$
\left\|S_{1}^{1 / 2} A_{2}^{*-\delta} v\right\|_{-\gamma} \leqslant C_{1}\|v\|_{0}
$$

даже для $v \in H_{0}$.

Кроме того, $B S_{1}^{1 / 2}$ - ограниченный оператор из $H_{1+\gamma}$ в $H_{\gamma}$ :

$$
\left\|B S_{1}^{1 / 2} u\right\|_{\gamma} \leqslant C_{2}\|u\|_{1+\gamma} .
$$


Далее, мы имеем с учетом того, что $A_{2}^{*}=A_{1}^{*}$ на $D\left(A_{2}^{*}\right)$,

$$
A_{2}^{*(1+\gamma) / 2} v=A_{2}^{*-\delta} A_{2}^{*} v=A_{2}^{*} A_{2}^{*-\delta} v=A_{1}^{*} A_{2}^{*-\delta} v .
$$

Поэтому $\left(u, A_{2}^{*(1+\gamma) / 2} v\right)_{0}=\left(A_{1} u, A_{2}^{*-\delta} v\right)_{0}$, или (см. (3.6))

$$
\left(A_{2}^{(1+\gamma) / 2} u, v\right)_{0}=\left(B S_{1}^{1 / 2} u, S_{1}^{1 / 2} A_{2}^{*-\delta} v\right)_{0} .
$$

Отсюда в силу обобщенного неравенства Шварца и неравенств (3.9), (3.10)

$$
\left|\left(A_{2}^{(1+\gamma) / 2} u, v\right)_{0}\right| \leqslant C_{3}\left\|B S_{1}^{1 / 2} u\right\|_{\gamma}\left\|S_{1}^{1 / 2} A_{2}^{*-\delta} v\right\|_{-\gamma} \leqslant C_{4}\|u\|_{1+\gamma}\|v\|_{0} .
$$

Так как $D\left(A_{2}^{*}\right)$ плотно в $H_{0}$, то получаем, что

$$
\left\|A_{2}^{(1+\gamma) / 2} u\right\|_{0} \leqslant C_{4}\|u\|_{1+\gamma}
$$

Это неравенство предельным переходом распространяется на $u \in H_{1+\gamma}$. Из него видно, что $H_{1+\gamma}$ содержится в $D\left(A_{2}^{(1+\gamma) / 2}\right)$ и что вложение непрерывно, поскольку левую часть в (3.11) можно считать нормой в $D\left(A_{2}^{(1+\gamma) / 2}\right)$.

Для $D\left(A_{2}^{*(1+\gamma) / 2}\right)$ все аналогично.

Доказательство леммы 3.3. Мы имеем теперь, используя первое равенство в (3.4) и теорему о реитерации (см. [9] или [17]),

$$
H_{1}=\left[H_{0}, H_{1+\gamma}\right]_{1 /(1+\gamma)} \subset\left[H_{0}, D\left(A_{2}^{(1+\gamma) / 2}\right)\right]_{1 /(1+\gamma)}=\left[H_{0}, D\left(A_{2}\right)\right]_{1 / 2}=D\left(A_{2}^{1 / 2}\right) .
$$

Аналогично получаем, что $H_{1} \subset D\left(A_{2}^{* 1 / 2}\right)$.

Этим лемма 3.3 и теорема 3.2 доказаны.

Перейдем к оператору $A_{1}$.

Теорема 3.5. При наших предположениях чисто мнимые степени оператора $A_{1}$ локалъно ограничены. При $0<\alpha<1$

$$
D\left(A_{1}^{\alpha}\right)=\left[H_{-1}, H_{1}\right]_{\alpha} .
$$

Этот результат следует из его эквивалентности результату теоремы 3.2, которая объяснена в [22, пп. 5.5.2 и 4.4.10]. Впрочем, эта эквивалентность проверяется несложно. Если $D\left(A_{2}^{1 / 2}\right)=H_{1}$, то

$$
\begin{aligned}
D\left(A_{1}^{1 / 2}\right) & =A_{1}^{-1 / 2} H_{-1}=A_{1}^{1 / 2} A_{1}^{-1} H_{-1}=A_{1}^{1 / 2} H_{1} \\
& =A_{1}^{1 / 2} A_{2}^{-1 / 2} A_{2}^{1 / 2} H_{1}=A_{1}^{1 / 2} A_{2}^{-1 / 2} H_{0}=H_{0} .
\end{aligned}
$$

Здесь равенство $A_{2}^{-1 / 2}=A_{1}^{-1 / 2}$ на $H_{0}$ следует из формулы вида $(2.2)$ с $\alpha=1 / 2$ для $A_{2}$ : в ней можно заменить $R(\lambda)=\left(A_{2}-\lambda I\right)^{-1}$ на $\left(A_{1}-\lambda I\right)^{-1}$, поскольку $A_{2}=A_{1}$ на $D\left(A_{2}\right)$. Этим равенство (3.12) получено для $\alpha=1 / 2$. На остальные $\alpha$ оно распространяется при помощи теоремы 6.6.8 из [35] (Хаасе указывает, что эта теорема принадлежит по существу Коматсу), в силу которой справедливость этого равенства следует из его справедливости для какого-нибудь значения $\alpha$. Объяснение обратной импликации ((3.12) для $\alpha=1 / 2 \Rightarrow(3.3))$ занимает у Арендта две строчки. Операторы $A_{1}^{1 / 2}$ и $A_{2}^{* 1 / 2}$ оказываются сопряженными в смысле теории ограниченных операторов в парах банаховых (у нас гильбертовых) пространств. 
Добавим также, что интерполяционные шкалы $\left\{H_{-1+2 \alpha}\right\}, 0 \leqslant \alpha \leqslant 1$, и $\left\{\left[H_{0}, D\left(A_{2}\right)\right]_{\theta}\right\}, 0 \leqslant \theta \leqslant 1$, «склеиваются» в одну шкалу по теореме Вольфа (см. [54] и [38]) благодаря положительному решению проблемы Като (так как имеет место совпадение этих шкал на пространствах $H_{0}$ и $H_{1}$ ). Поэтому операторы $A_{1}$ и $A_{2}$ оказываются «согласованными» в пространствах между $H_{-1}$ и $D\left(A_{2}\right)$. Любое утверждение о гладкости для решений уравнения $A_{1} u=f$ дает некоторую информацию о $D\left(A_{2}\right)$. См. [48] и [1]-[3] или [7]. И наоборот, любая информация о $D\left(A_{2}\right)$ может содержать утверждение о гладкости для решений уравнения $A_{1} u=f$.

\section{4. Некоторые приложения.}

4.1. Смешанные и более общие задачи. Смешанная задача для системы $L u=f$ состоит в том, что ищется решение, подчиненное однородным условиям Дирихле на части $\Gamma_{1}$ границы и однородным условиям Неймана на дополнительной части $\Gamma_{2}$. Смешанным задачам посвящена очень большая литература. В [5], [7] рассмотрен случай, когда $\Gamma_{1}$ - область на замкнутой липшицевой поверхности с липшицевым краем. Пространство $H_{1}$ - это подпространство $H_{\Gamma_{1}}^{1}(\Omega)$ в $H^{1}(\Omega)$, состоящее из функций, равных нулю на $\Gamma_{1}$. Нужна сильная коэрцитивность формы (0.3) на этом пространстве (вытекающая из сильной коэрцитивности на $H^{1}(\Omega)$ ). Отдельно рассматривать соответствующие операторы $A_{1}$ и $A_{2}$ не приходится (ср. [45], [30]), применимы результаты из п. 3. Результаты обобщаются на случай, когда $\Gamma_{1}$ и $\Gamma_{2}$ - открытые множества, состоящие из компонент связности с липшицевыми краями.

Отметим, что здесь нужен следующий результат об интерполяции для пространств $H_{\Gamma_{1}}^{s}(\Omega)$ : если $1-\varepsilon<s_{1}<s_{2}<1+\varepsilon$, то

$$
\left[H_{\Gamma_{1}}^{s_{1}}(\Omega), H_{\Gamma_{1}}^{s_{2}}(\Omega)\right]_{\theta}=H_{\Gamma_{1}}^{(1-\theta) s_{1}+\theta s_{2}}(\Omega) \quad(0<\theta<1) .
$$

Укажем простой способ его получения в предположении однозначной разрешимости задачи Дирихле для рассматриваемых $s$. Пространство $H^{s}(\Omega)$ можно представить в виде прямой суммы подпространства $\widetilde{H}^{s}(\Omega)$ и подпространства решений однородной системы $L u=0$ («разложение Вейля», см. [6], [7]). Последнее параметризуется пространством $H^{s-1 / 2}(\Gamma)$ данных Дирихле этих решений. Аналогичное разложение имеет место для $H_{\Gamma_{1}}^{s}(\Omega)$, только второе подпространство надо заменить пространством решений однородной системы с нулевыми данными Дирихле на $\Gamma_{1}$. Последнее параметризуется пространством $\widetilde{H}^{s-1 / 2}\left(\Gamma_{2}\right)$ данных Дирихле этих решений - функций из $H^{s-1 / 2}(\Gamma)$ с носителями на $\bar{\Gamma}_{2}$. Интерполяция пространств $\widetilde{H}^{s}(\Omega)$ и $\widetilde{H}^{s-1 / 2}\left(\Gamma_{2}\right)$ не вызывает проблем. Отметим, что дело обстоит сложнее при более общем, чем у нас, крае $\partial \Gamma_{1}$, cp. [33].

Можно рассмотреть и более общие задачи, в которых область определения оператора $A_{1}$ - некоторое подпространство в $H^{1}(\Omega)$, содержащее $\stackrel{\circ}{H^{1}}(\Omega)$. Cp., например, [45]. Но здесь нам надо предположить, что выполнено наше условие 3.1. Тогда снова применимы результаты из п. 3.

4.2. Операторы на липшицевой границе. Теоремы 3.2 и 3.5 приложимы также $\kappa$ операторам на липшицевой гранище $\Gamma$, а именно, к оператору Дирихле $D$, к оператору $A^{-1}$ (где $A \psi$ - сужение на границу потенциала простого слоя 
$\mathscr{A} \psi)$ и к гиперсингулярному оператору $H$ (см. [3], [6], [7]) при уточняемых ниже предположениях.

Оператор Дирихле - это оператор, переводящий данные Дирихле для решений однородной системы $L u=0$ в данные Неймана. Ему посвящена большая литература. В [3], [7] он рассмотрен для систем 2-го порядка в ограниченной липшицевой области.

Для введения операторов $A$ и $H$ удобно иметь две области $\Omega^{ \pm}$с общей границей. В [3], [7] рассмотрен случай, когда они вместе с их общей липшицевой границей составляют стандартный тор $\mathbb{T}^{n}$.

Во всех этих случаях $H_{1}=H^{1 / 2}(\Gamma), H_{0}=H^{0}(\Gamma)$ и $H_{-1}=H^{-1 / 2}(\Gamma)$. В «гладких» задачах $H_{2}=H^{1}(\Gamma)$.

В частности, формула (3.12) справедлива для оператора Дирихле как оператора из $H^{1 / 2}(\Gamma)$ в $H^{-1 / 2}(\Gamma)$. В общих негладких задачах $D\left(A_{2}\right)$ остается неизвестной, хотя некоторая информация о $D\left(A_{2}\right)$ получается из теорем о гладкости (см. [48] и [3], [7]).

Во всех случаях граничных операторов первичны не форма, а оператор, по которому определяется форма:

$$
\Phi(u, v)=\left(A_{1} u, v\right)_{0} .
$$

Ее сильная коэрцитивность в случае оператора $D$ следует из сильной коэрцитивности формы $(0.3)$ на $H^{1}(\Omega)$. В случае оператора $A^{-1}$ достаточно иметь сильную коэрцитивность формы вида $(0.3)$ на $H^{1}\left(\mathbb{T}^{n}\right)$, а в случае оператора $H$ - на $H^{1}\left(\Omega^{ \pm}\right)$. См. [3], [7] и приведенные там ссылки. Снова не требуется отдельное рассмотрение этих ситуаций, применимы результаты из п. 3.

Эти результаты можно перенести и на рассмотренные в [4], [5], [7] операторы на части липшицевой границы с липшицевым краем. Можно охватить и фредгольмовы ситуации.

4.3. Задачи для систем высших порядков. Пусть $m_{1}, \ldots, m_{d}$ - заданные целые положительные числа. Сильную эллиптичность можно определить для матричного оператора $L=\left(L_{r, s}\right)_{r, s=1}^{d}$, где ord $L_{r, s}=m_{r}+m_{s}$. См. [50], где рассмотрена задача Дирихле с решениями из $\widetilde{H}^{m}(\Omega),[49]$ и [2], где рассмотрена и задача Неймана. Как и раньше, $\Omega$ - ограниченная липшицева область. Здесь

$$
H^{\boldsymbol{m}}(\Omega)=\prod_{i=1}^{d} H^{m_{i}}(\Omega), \quad \widetilde{H}^{\boldsymbol{m}}(\Omega)=\prod_{i=1}^{d} \widetilde{H}^{m_{i}}(\Omega) .
$$

Например, в задаче Неймана $H_{1}=H^{m}(\Omega)$ и $H_{-1}=\prod_{i=1}^{d} \widetilde{H}^{-m_{i}}(\Omega)$. Форма $\Phi(u, v)$ имеет вид

$$
\int_{\Omega} \sum_{j, k=1}^{d} \sum_{|\alpha|=m_{j},|\beta|=m_{k}} a_{\alpha, \beta}^{j, k} \partial^{\beta} u_{k}(x) \cdot \partial^{\alpha} \overline{v_{j}(x)} d x+\ldots
$$

Как и для систем 2-го порядка, в случае задачи Дирихле коэрцитивность следует из сильной эллиптичности, а в случае задачи Неймана является добавочным условием; достаточные условия см., например, в [49] и [2]. По крайней мере, эти задачи не требуют дополнительных рассмотрений, применимы теоремы из п. 3. Cp. [26]. 
5. Сравнение нашего подхода с известными. Как мы уже упоминали, исследованию проблемы Като посвящена обширная литература. Давно были получены полные результаты для задач Дирихле и Неймана в липшицевых областях, см., например, [47], [24], [36] и приведенные там ссылки. Важнейшие результаты принадлежат Аушеру-Чамитчану, Макинтошу и другим математикам. См. наш список литературы, но он далеко не полон; см. также ссылки в работах этого списка. Терминология в этих работах, как правило, не совпадает с нашей, но объясняется, так что эти расхождения не затруднят читателя.

В этих работах главным образом рассматривается оператор $A_{2}$, хотя оператор $A_{1}$ и упомянутые в п. 3 эквивалентности были известны. В нашем же подходе очень существенно используются возможность одновременного рассмотрения этих двух операторов и некоторые специфические свойства оператора $A_{1}$. При помощи оператора $A_{1}$ решается проблема Като для оператора $A_{2}$, что, в свою очередь, влечет нужные результаты для оператора $A_{1}$. По-видимому, наш подход заслуживает внимания благодаря простоте и единообразию рассмотрений.

$\mathrm{B}$ частности, нам удалось избежать рассмотрений сначала в $\mathbb{R}^{n}$, полупространстве и неограниченных «специальных» липшицевых областях. Этот путь используется в [27], [28], см. также ссылки в этих работах.

Мы могли включить в рассмотрение случай операторов в $\mathbb{R}^{n}$, отказавшись от предположения о компактности вложений (1.1). Можно было бы рассмотреть и задачи в неограниченных (строго) липшицевых областях. Но в таких областях интересны различные предположения о поведении решений на бесконечности, влияющие на выбор пространств, и мы сочли, что кратко останавливаться на этом нецелесообразно.

Как в [45] и [32], нам нужна небольшая гладкость старших коэффициентов в (0.1). Достаточно предполагать, что это мультипликаторы в пространствах $H^{s}(\Omega)$ с малыми $|s|$. Младшие коэффициенты можно считать ограниченными и измеримыми в $\Omega$. «Средние» коэффициенты можно было бы предположить липшицевыми, чтобы иметь возможность дифференцировать их при переходе к формально сопряженной системе. Но экономнее рассматривать наш оператор как слабое возмущение оператора без средних и младших членов, как, например, в [32]. Тогда и средние коэффициенты можно считать ограниченными и измеримыми. Аналогично обстоит дело с задачами для систем высших порядков. В некоторых работах (см., например, [30], [23]) положительное решение проблемы Като в рассмотренных там ситуациях получено в предположении ограниченности и измеримости старших коэффициентов.

Далее, случай системы (0.1) для нас не сложнее случая скалярного уравнения. Нам не пришлось имитировать структуру скалярного уравнения в дивергентной форме $\operatorname{div}(a(x) \nabla) u=f$ или строить сложную факторизацию в матричном случае: по существу нам хватило факторизации (3.6). Работа [32] подсказала нам идею доказывать включения (3.8), но ее предположения обременительны в случае матричных $L$.

Мы не упоминали $H^{\infty}$-ограниченное функциональное исчисление (см., в частности, [46], [25]); на самом деле его наличие для $A_{1}$ эквивалентно справедливости теорем 3.2 и 3.5, см. [22], и это содержательный факт.

Мы не останавливались на обобщениях предположений о сомножителях в (3.6), при которых можно провести наше доказательство теоремы 3.2.

Мы не затрагивали обобщения на случай пространства $L_{p}$ вместо $L_{2}$. Глубокие результаты в этом направлении получены, в частности, в [27], [29], [23], [37]. 
По-видимому, в конкретных ситуациях можно рассчитывать на экстраполяцию результатов на $p$, близкие к 2 (ср. [31]).

Вопросы разрешимости упомянутых выше задач и уравнений с оператором $A_{1}$, гладкости решений и спектральные свойства соответствующих операторов $A_{1}$ в липшицевых областях и на липшицевых поверхностях рассмотрены первым из авторов в работах [1]-[6]. Этот материал вошел в книгу [7]. В нее удалось успеть включить основные результаты настоящей статьи, они были получены совместно. Второй автор вслед за [16], [53], [19] доказал равенство (3.3) для дифференциально-разностных операторов при существенных ограничениях на геометрию области (см. [14], [15]); его доказательство в случае липшицевой границы, использующее метод, изложенный в данной работе, готовится к публикации.

Авторы искренне благодарны А. Л. Скубачевскому и Т. А. Суслиной: они прочитали рукопись и сделали очень ценные замечания.

\section{ЛиТЕРАТУРА}

[1] М. С. Агранович, Регулярность вариащионных решений линейных граничных задач в липшицевых областях, Функц. анализ и его прил., 40:4 (2006), 83-103.

[2] М. С. Агранович, K теории задач Дирихле и Неймана для линейных сильно эллиптических систем в липшицевых областях, Функц. анализ и его прил., 41:4 (2007), 1-21.

[3] М. С. Агранович, Операторы типа потенииала и задачи сопряжения для силъно эллиптических систем 2-го порядка в областях с липшищевой гранищей, Функц. анализ и его прил., 43:3 (2009), 3-25.

[4] М. С. Агранович, Силъно эллиптические системы 2-го порядка с граничными условиями на незамкнутой липшищевой поверхности, Функц. анализ и его прил., 45:1 (2011), 1-15.

[5] М. С. Агранович, Смешанные задачи в липшищевой области для силъно эллиптических систем 2-го порядка, Функц. анализ и его прил., 45:2 (2011), 1-22.

[6] M. S. Agranovich, Remarks on strongly elliptic second-order systems in Lipschitz domains, Russian J. Math. Phys., 20:4 (2012), 405-416.

[7] М. С. Агранович, Соболевские пространства, их обобщения и эллиптические задачи в областях с гладкой и липшищевой гранищей, Изд. МЦНМО, М., 2013.

[8] М. С. Агранович, М. И. Вишик, Эллиптические задачи с параметром и параболические задачи общего вида, УМН, 19:3 (1964), 53-161.

[9] Й. Берг, Й. Лёфстрём, Интерполячионные пространства, Мир, М., 1980.

[10] М. И. Вишик, О сильно эллиптических системах дифференциальных уравнений, Матем. сб., 29(71):3 (1951), 615-676.

[11] Т. Като, Теория возмущений линейных операторов, Мир, М., 1972.

[12] М. А. Красносельский, П. П. Забрейко, Е. И. Пустыльник, П. Е. Соболевский, Интегральные операторы в пространствах суммируемых функиий, Наука, М., 1966.

[13] Ж.-Л. Лионс, Э. Мадженес, Неоднородные граничные задачи и их приложения, Мир, М., 1971.

[14] А. М. Селицкий, Пространство начальных данных 3-й краевой задачи для параболического дифберенииально-разностного уравнения в одномерном случае, Матем. заметки, 92:4 (2012), 636-640.

[15] А. М. Селицкий, Моделирование некоторых оптических систем на основе параболического дифференииально-разностного уравнения, Матем. моделирование, 24:12 (2012), 38-42. 
[16] А. Л. Скубачевский, Р. В. Шамин, Параболические дифференииалъно-разностные уравнения второго порядка, Докл. РАН, 379:5 (2001), 595-598.

[17] Х. Трибель, Теория бункииональных пространств, Мир, М., 1986.

[18] Л. Хёрмандер, Линейные дифференииалъные операторы с частными производными, Мир, М., 1965.

[19] Р. В. Шамин, О пространствах начальных данных для дифференциалъных уравнений в гилъбертовом пространстве, Матем. сб., 194:9 (2003), 141-156.

[20] И. Я. Шнейберг, Спектральные свойства линейных операторов в интерполяиионных семействах банаховых пространств, Матем. исслед., 9:2 (1974), 214-229.

[21] Sh. Agmon, On the eigenfunctions and on the eigenvalues of general elliptic boundary value problems, Comm. Pure Appl. Math., 15:2 (1962), 119-147.

[22] W. Arendt, Semigroups and evolution equations: functional calculus, regularity and kernel estimates, in: Handbook of Differential Equations, Evolutionary Differential Equations, vol. 1, Elsevier/North-Holland, Amsterdam, 2004, 1-85.

[23] P. Auscher, N. Badr, R. Haller-Dintelmann, J. Rehberg, The square root problem for second order, divergence form operators with mixed boundary conditions on $L^{p}$, http://arxiv.org/abs/1210.0780v1.

[24] P. Auscher, S. Hofmann, M. Lacey, J. Lewis, A. McIntosh, P. Tchamitchian, The solution of Kato's conjectures, C. R. Acad. Sci. Paris, Sér. 1, 332:7 (2001), 601-606.

[25] P. Auscher, A. McIntosh, A. Nahmod, Holomorphic functional calculi of operators, quadratic estimates and interpolation, Indiana Univ. Math. J., 46:2 (1997), 375-403.

[26] P. Auscher, S. Hofmann, A. McIntosch, P. Tchamitchian, The Kato square root problem for higher order elliptic operators and systems on $\mathbb{R}^{n}$, J. Evol. Equ., 1:4 (2001), 361385.

[27] P. Auscher, P. Tchamitchian, Square root problem for divergence operators and related topics, Astérisque, 249 (1998), 1-171.

[28] P. Auscher, P. Tchamitchian, Square roots of elliptic second order divergence operators on strongly Lipschitz domains: $L^{2}$ theory, J. Anal. Math., 90 (2003), 1-12.

[29] P. Auscher, P. Tchamitchian, Square roots of elliptic second order divergence operators on strongly Lipschitz domains: $L^{p}$ theory, Math. Ann., 320:3 (2001), 577-623.

[30] A. Axelsson, S. Keith, A. McIntosh, The Kato square root problem for mixed boundary value problems, J. London Math. Soc., 74:1 (2006), 113-130.

[31] S. Blunck, P. Kunstmann, Calderón-Zygmund theory for non-integral operators and the $H^{\infty}$ functional calculus, Rev. Mat. Iberoamericana, 19:3 (2003), 919-942.

[32] A. F. M. ter Elst, D. W. Robinson, On Kato's square root problem, Hokkaido Math. J., 26:2 (1997), 365-376.

[33] J. Griepentrog, K. Gröger, H.-Ch. Kaiser, J. Rehberg, Interpolation for function spaces related to mixed boundary value problems, Math. Nachr., 241 (2002), 110-120.

[34] D. Grisvard, Caractérisation de quelques espaces d'interpolation, Arc. Rational Mech. Anal., 25 (1967), 40-63.

[35] M. Haase, The Functional Calculus for Sectorial Operators, Birkhäuser, Basel, 2006.

[36] S. Hoffmann, A short course on the Kato problem, Contemp. Math., 289 (2001), 61-67.

[37] T. Hytönen, A. McIntosh, P. Portal, Kato's square root problem in Banach spaces, J. Funct. Anal., 254:3 (2008), 675-726.

[38] S. Janson, P. Nilsson, J. Peetre, Notes on Wolff's note on interpolation spaces, Proc. London Math. Soc. (3), 48:2 (1984), 283-299.

[39] T. Kato, Fractional powers of dissipative operators, J. Math. Soc. Japan, 13 (1961), 246-274.

[40] T. Kato, Fractional powers of dissipative operators, II, J. Math. Soc. Japan, 14 (1962), $242-248$.

[41] H. Komatsu, Fractional powers of operators, Pacif. J. Math., 19 (1966), 285-346. 
[42] J. L. Lions, Équations différentielles operationelles et problèmes aux limites, SpringerVerlag, Berlin etc., 1961.

[43] J. L. Lions, Espaces d'interpolation et domaines de puissances fractionaires d'opeŕateurs, J. Math. Soc. Japan, 14 (1962), 233-241.

[44] A. McIntosh, On the compatibility of $A^{1 / 2}$ and $A^{* 1 / 2}$, Proc. Amer. Math. Soc., 32:2 (1972), 430-434.

[45] A. McIntosh, Square roots of elliptic operators, J. Funct. Anal., 61:3 (1985), 307-327.

[46] A. McIntosh, Operators which have an $H^{\infty}$ functional calculus, in: Miniconference on Operator Theory and Partial Differential Equations, Proc. Centre Math. Anal. Austral. Nat. Univ., vol. 14, Austral. Nat. Univ., Canberra, 1986, 210-231.

[47] A. McIntosh, The Square Root Problem for Elliptic Operators: a Survey, Lecture Notes in Math., vol. 1450, Springer-Verlag, Berlin, 1990.

[48] W. McLean, Strongly Elliptic Systems and Boundary Integral Equations, Cambridge Univ. Press, Cambridge, UK, 2000.

[49] J. Nečas, Les méthodes directes en théorie des equations elliptiques, Masson, Paris, 1967; Direct Methods in the Theory of Elliptic Equations, Springer-Verlag, BerlinHeidelberg, 2012.

[50] L. Nirenberg, Remarks on strongly elliptic partial differential equations, Comm. Pure Appl. Math., 8 (1965), 649-675.

[51] R. T. Seeley, Norms and domains of the complex powers $A_{B}^{z}$, Amer. J. Math., 93:2 (1971), 299-309.

[52] R. T. Seeley, Interpolation in $L_{p}$ with boundary conditions, Studia Math., 44 (1972), 47-60.

[53] A. L. Skubachevskii, R. V. Shamin, The mixed boundary value problem for parabolic differential-difference equation, Funct. Differ. Eq., 8:3-4 (2001), 407-424.

[54] T. W. Wolff, A note on interpolation spaces, in: Lecture Notes in Math., vol. 918, Springer-Verlag, Berlin-New York, 1982, 199-204.

[55] A. Yagi, Coincidence entre des espaces d'interpolation et des domaines de puissances fractionaires d'opreateurs, C. R. Acad. Sci. Paris, Sér. 1, 299:6 (1984), 173-176.

Московский институт электроники и математики

Национальный исследовательский университет

«Высшая школа экономики»

e-mail: magran@orc.ru

Вычислительный центр РАН им. А. А. Дородницына

e-mail: selitsky@mail.ru
Поступило в редакцию 17 января 2013 г. 